This document is the unedited Author's version of a Submitted Work that was subsequently accepted for publication in 'Bioconjugate Chemistry', copyright (C) 2019 American Chemical Society after peer review. To access the final edited and published work see https://doi.org/10.1021/acs.bioconjchem.9b00248

\title{
A covalently attached progesterone molecule outcompetes the binding of free progesterone at an allosteric site of cytochrome P450 3A4
}

\author{
Julie Ducharme, Vanja Polic, Karine Auclair* \\ Department of Chemistry, McGill University, Montreal, Quebec, Canada H3A 0B8 \\ *Corresponding author: karine.auclair@mcgill.ca; tel: +1-514-398-2822; fax: +1-514-398-3797 \\ Keywords: P450, CYP3A4, allostery, bioconjugation, progesterone
}

\begin{abstract}
Because of its exceptional substrate promiscuity, human P450 3A4 (CYP3A4) is arguably the most important drug-metabolizing enzyme. CYP3A4 also has the particularity of binding multiple ligands simultaneously, which is associated with heterotropic or homotropic, positive or negative, cooperativity or allostery. Solving the kinetics of such complex systems remains challenging, and so is identifying the binding pockets involved. Progesterone (PRG) is a known allosteric activator of CYP3A4-catalyzed 7-benzyloxy-4-trifluoromethylcoumarin (BFC) debenzylation. We report herein the use of bioconjugation as a successful strategy to identify this PRG allosteric site. A progesterone analogue (PGM) was covalently attached, separately at several locations, near a peripheral binding pocket previously proposed to be an allosteric site. Studies of BFC debenzylation in the presence of free PRG revealed that two of the bioconjugates successfully positioned the covalently attached PGM moiety in a way that mimcs the allosteric activation observed with free PRG. Interestingly, the PGM bioconjugate with the better fit yielded a higher permanent activation of the enzyme.
\end{abstract}

\section{Introduction}

Allostery is a key biological phenomenon and has remained a central research focus owing to its fundamental role in essential functions (e.g. cellular signaling) and in diseases. ${ }^{1,2}$ Cytochrome P450 enzymes (CYPs), which are hemedependent monooxygenases, are also known to be regulated by allosteric mechanisms. Drug metabolizing CYPs in particular, often have several known positive and/or negative allosteric effectors, which may lead to drug interactions. In humans, CYP3A4 is responsible for the metabolism of approximately $50 \%$ of all commercially available drugs. ${ }^{3}$ The unequalled substrate promiscuity of CYP3A4 is attributed to its large and flexible active site, which can bind ligands of different sizes and shapes, and can even bind multiple ligands simultaneously. ${ }^{4}$ Investigating the kinetics of CYP3A4 remains difficult as the multiple ligands binding events lead to complex cooperative and allosteric effects. For instance, positive or negative homotropic cooperativity has been described with substrates such as testosterone, aflatoxin B1, $17 \beta$-estradiol, amitriptyline, and progesterone. ${ }^{5,6}$ Moreover, some substrates of CYP3A4 also act as effectors in the transformation of other substrates, a phenomenon described as heterotopic cooperativity. Progesterone (PRG) for instance is a known activator of CYP3A4-catalyzed 7-benzyloxy-4-trifluoromethylcoumarin (BFC) debenzylation ${ }^{7}$, while $\alpha$-naphthoflavone activates testosterone metabolism, and testosterone ${ }^{8}$ can increase the turnover of the anxiolytic drug midazolam. ${ }^{9}$ 
This document is the unedited Author's version of a Submitted Work that was subsequently accepted for publication in 'Bioconjugate Chemistry', copyright (C) 2019 American Chemical Society after peer review. To access the final edited and published work see https://doi.org/10.1021/acs.bioconjchem.9b00248

In an effort to explain the sigmoidal enzyme kinetics observed with various substrates and effectors, an allosteric mechanism was postulated for CYP3A4 35 years ago. ${ }^{10-12}$ This hypothesis was greatly reinforced when the first CYP3A4 crystal structure was published by William et al. in 2004, revealing an unexpected peripheral binding site for PRG, proposed to be an allosteric pocket (Figure 1). ${ }^{13}$ Since then, many publications ${ }^{14-25}$ have reported results that are consistent with this PRG binding site being an allosteric site, yet its exact location is still questioned as the identified PRG binding pocket may result from a crystallographic artifact. Several steroids are known to act as allosteric effectors of CYP3A4, often in a substrate-dependent manner. ${ }^{26}$ Since steroids are widely distributed in vivo, their impact on CYP3A4 can have serious implications for drug metabolism.

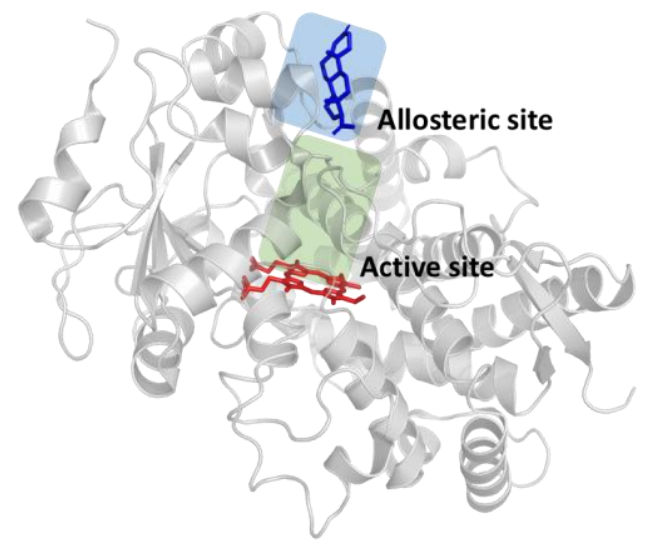

Figure 1. Crystal structure of P450 3A4 with co-crystallized PRG in the putative allosteric site (PDB: 1WOF). The approximate locations of the allosteric site and the active site are represented by a blue and green box respectively.

CYP3A4 hydroxylates BFC at the benzylic position, eventually leading to the formation of 7-hydroxy-4trifluoromethylcoumarin (HFC). ${ }^{27}$ This enzymatic reaction is accelerated with the addition of PRG to the mixture, likely via PRG binding to an allosteric pocket. We report here the use of bioconjugation to narrow down the location of this allosteric site. We have previously reported that the covalent attachment of a PRG analogue (PGM) at the peripheral binding site observed in the crystal structure of CYP3A4 leads to a large increase in the catalytic rate of the enzyme. ${ }^{28,29}$ Herein, further studies with this bioconjugate and several new ones demonstrate that covalently linking PGM to CYP3A4 can efficiently mimic the allosteric effect of PRG and can also reveal other mechanisms of activation.

\section{$\underline{\text { Result and Discussion }}$}

\section{Design of CYP3A4 mutants}

Regioselective bioconjugation of CYP3A4 was achieved using maleimide-cysteine chemistry as previously reported. ${ }^{28,30}$ Therefore, mutants containing a single reactive cysteine were generated from a parent cysteine-depleted mutant (mut0) ${ }^{28}$ with only two remaining cysteines (Table 1). One of these cysteines (C442) is the heme iron ligand, while the other (C377) is buried and unreactive. ${ }^{30}$ The mutation sites were selected based on visual inspection of the CYP3A4 crystal structure with bound PRG (PDB:1WOF) ${ }^{13}$ to seek residues in proximity to the putative allosteric pocket 
This document is the unedited Author's version of a Submitted Work that was subsequently accepted for publication in 'Bioconjugate Chemistry', copyright (C) 2019 American Chemical Society after peer review. To access the final edited and published work see https://doi.org/10.1021/acs.bioconjchem.9b00248

(Figure S1). The target residues were selected to be well distributed around the co-crystallized PRG in order to probe different binding locations and orientations for PGM (Figure 2). Of the 11 mutants designed to contain a single reactive cysteine, 9 were successfully expressed and purified (mut1 - mut9) and their activity towards BFC debenzylation was confirmed (Figure S2).

\begin{tabular}{l|l} 
Mutant name & Mutations \\
\hline WT & $\Delta 1-10$ \\
\hline Mut0 & C58T/ C64A/ C98S/ C239S/ C468G \\
\hline Mut1 & Mut0 + F108C \\
\hline Mut2 & Mut0 + G481C \\
\hline Mut3 & Mut0 + L482C \\
\hline Mut4 & Mut0 + V111C \\
\hline Mut5 & Mut0 + Q484C \\
\hline Mut6 & Mut0 + F215C \\
\hline Mut7 & Mut0 + I301C \\
\hline Mut8 & Mut0 + F304C \\
\hline Mut9 & Mut0 + L210C \\
\hline Mut10 & Mut0 + I120C \\
\hline Mut11 & Mut0 + G109C
\end{tabular}

Table 1. P450 3A4 mutants. Although Mut0 was generated from the truncated WT enzyme, residue numbering is in reference to the full-length P450 3A4 sequence

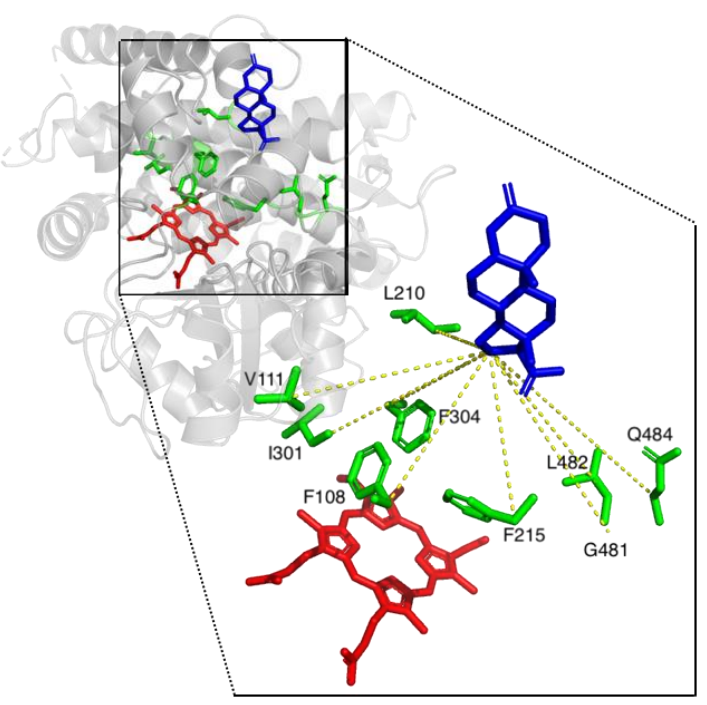

Figure 2. Crystal structure of P450 3A4 with co-crystallized PRG in blue and heme in red (PDB: 1WOF). Highlighted in green are the residues that were mutated to cysteine in the single-cysteine mutants, and designed to be different attachment points of the PGM label. Mutated residues are located either around the active site or the putative allosteric pocket of CYP3A4, yet not on the protein surface, and with the expectation that the PGM label may reach the allosteric pocket.

\section{Accessibility of the newly introduced cysteine residues}


This document is the unedited Author's version of a Submitted Work that was subsequently accepted for publication in 'Bioconjugate Chemistry', copyright (C) 2019 American Chemical Society after peer review. To access the final edited and published work see https://doi.org/10.1021/acs.bioconjchem.9b00248

To investigate the accessibility of the newly introduced cysteine residues for bioconjugation, we looked at their reactivity. Thus, mut1, mut2 and mut8 were separately bioconjugated to several different maleimide-containing reagents (Figures S3, S4), before proceeding with the ligand analogue of interest, PGM, and mut1-8 (Figure S5). As expected, the bioconjugation yield was found to depend on the position of the cysteine. Under identical conditions, a lower bioconjugation yield was interpreted as the maleimide reagent having poor accessibility to the cysteine residue, or not being able to adopt the required orientation for the reaction to take place. On the other hand, a higher bioconjugation yield suggests a better accessibility of the cysteine, and potentially higher affinity of the maleimide derivative for the pocket near the cysteine. The doubly-labeled mutants were attributed to the presence of denatured protein exposing C377 and/or C442. Therefore, all mutants with low bioconjugation yields (mut7 and mut8) were not pursued further. PGM-bioconjugated mut4 was produced in only $42 \%$ yield but was nevertheless kept for further studies because preliminary studies showed a significant impact of PGM-bioconjugation on its activity. In contrast, mut9 was not pursued because PGM bioconjugation inactivated the enzyme (results not shown). Furthermore, the absorbance spectra of mut1, mut2 and mut4, with and without PGM bioconjugation, were obtained to investigate how labeling affects the CYP3A4 spin state (Figure S6).

\section{Enzyme activity of bioconjugates towards BFC}

Michaelis-Menten kinetics data was obtained for mut1-6, with and without PGM bioconjugation (Figure 3, Table 2). Except for mut2 and mut5, all bioconjugates showed higher $k_{\text {cat }}$ than the corresponding unmodified mutants. Mut 6 was the most activated $(\sim 4.5 \times)$, followed by mut1 and mut3 $(\sim 2 \times)$. In contrast, the $k_{\text {cat }}$ of mut2 and mut5 decreased after PGM bioconjugation, suggesting that the PGM label might be oriented in an unnatural direction, and detrimental to catalysis. The $\mathrm{K}_{\mathrm{M}}$ values were decreased after PGM bioconjugation for all mutants, consistent with a higher affinity for BFC. Furthermore, the specificity constant, $k_{\mathrm{cat}} / \mathrm{K}_{\mathrm{M}}$, was significantly enhanced for the bioconjugates of mut6 $(\sim 8 \times)$, mut4 $(4 \times)$, mut1 $(3 \times)$ and mut3 $(\sim 3 \times)$. The improvement observed with PGM bioconjugation on mut4 is largely the result of a decreased $\mathrm{K}_{\mathrm{M}}$. From these kinetic data, it appears that the enzyme is sensitive to the position of the PGM moiety. Although the newly introduced cysteine of mut2 (G481C) and of mut3 (L482C) are next to each other on a loop, PGM bioconjugation to either mutant affects $k_{\text {cat }}$ very differently, whereas PGM bioconjugation to mut2 (G481C) or mut5 (Q484C) has a similar effect. This data set further confirms that attaching PGM near the putative allosteric site of CYP3A4 can activate the enzyme and that the position and/or orientation of the PGM label can modulate enzyme activity. There may however be more than one allosteric pocket and from these results alone, it is not possible to confirm that the PGM moiety of these bioconjugates occupies the same allosteric site as free PRG does during the activation of CYP3A4 towards BFC debenzylation. ${ }^{7}$ To answer this question we next looked at the activation of our CYP3A4 variants and bioconjugates by free PRG.

\section{Impact of covalently-linked PGM on the activation of CYP3A4 by free PRG}

To verify if the PGM moiety of our bioconjugates occupies the same allosteric pocket as PRG, we investigated the BFC-debenzylation activity of our mutants and bioconjugates in the presence of free PRG. Enzyme-catalyzed BFC debenzylation was monitored directly by fluorescence, at a constant concentration of BFC, and at various amounts of PRG. As PRG is also a CYP3A4 substrate, the ratio of BFC to PRG had to be large enough to minimize PRG hydroxylation. 
This document is the unedited Author's version of a Submitted Work that was subsequently accepted for publication in 'Bioconjugate Chemistry', copyright (C) 2019 American Chemical Society after peer review. To access the final edited and published work see https://doi.org/10.1021/acs.bioconjchem.9b00248

Consistent with previous studies, ${ }^{28}$ PRG was found to activate WT CYP3A4. The optimal PRG concentration range to observe this activation was found to be $0-25 \mu \mathrm{M}$, at $50 \mu \mathrm{M}$ BFC with $0.7 \mu \mathrm{M}$ enzyme (Figure S7). Next, PRG activation profiles were obtained for mut1-6 before and after PGM bioconjugation (Figures 4 and S8). For all the unlabeled mutants, the PRG activation was conserved suggesting that the allosteric site of these mutants remains intact.
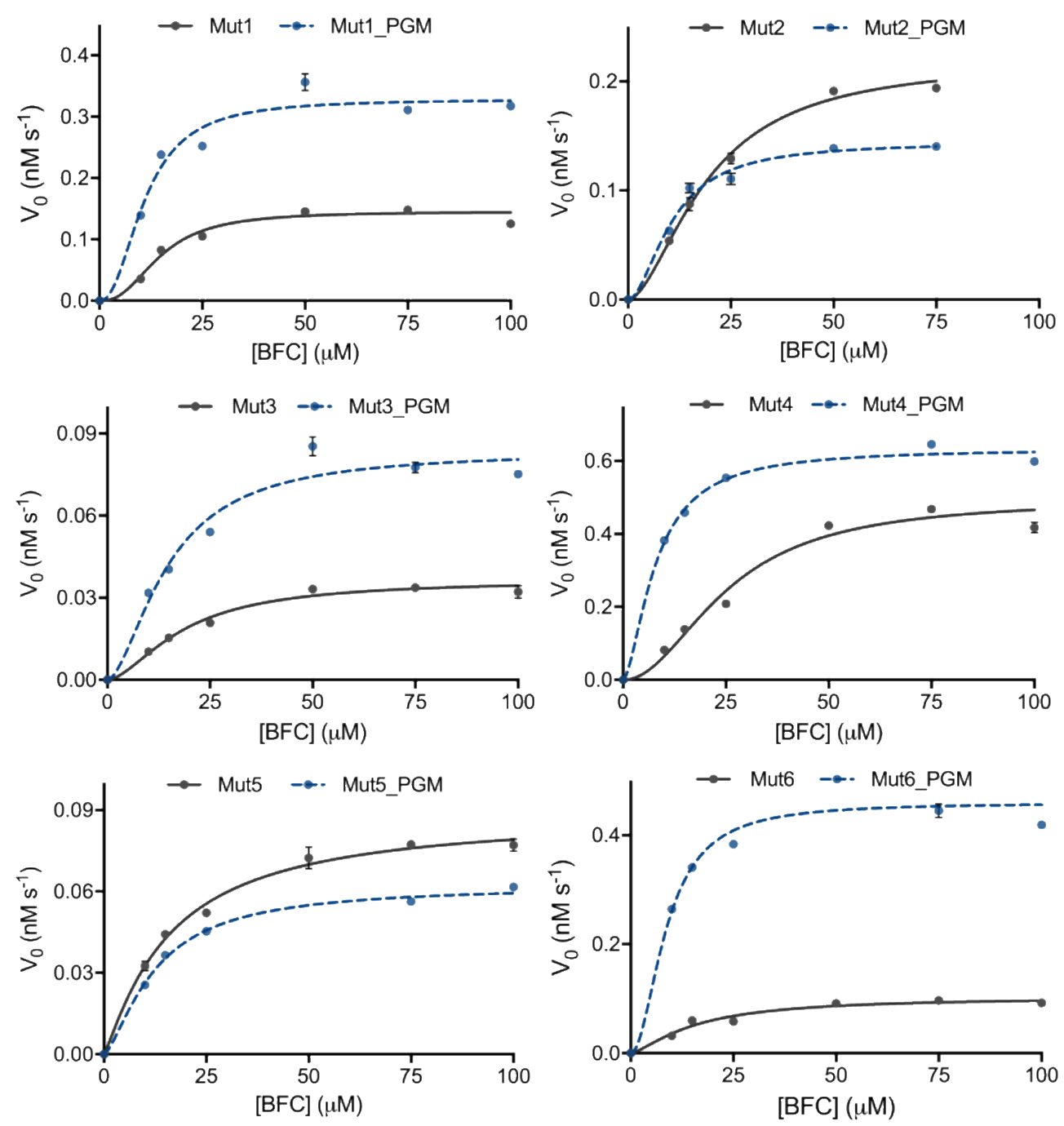

Figure 3. Michaelis-Menten kinetic assay comparison of PGM-labeled vs. unlabeled P450 3A4 mutants. The reactions contained $0.7 \mu \mathrm{M}$ P450, $0.7 \mu \mathrm{M}$ CPR, $0-100 \mu \mathrm{M}$ BFC and $1 \mathrm{mM}$ NADPH. The dotted blue curves represent the PGM-labeled mutants and the solid curves the unlabeled mutants. Each data point is the average of triplicates with the standard error shown with in error bars. The data points were curve fitted using the Hill equation (Figure S14). Refer to Figure S12 for Hill coefficients. 
This document is the unedited Author's version of a Submitted Work that was subsequently accepted for publication in 'Bioconjugate Chemistry', copyright (C) 2019 American Chemical Society after peer review. To access the final edited and published work see https://doi.org/10.1021/acs.bioconjchem.9b00248

\begin{tabular}{l|c|c|c|c} 
Mutants & $\mathrm{K}_{\mathrm{M}}^{\mathrm{a}, \mathrm{b}}$ & $k_{\text {cat }}{ }^{\mathrm{N}}$ & $k_{\text {cat }} / \mathrm{K}_{\mathrm{M}}{ }^{\mathrm{d}}$ & Fold $^{\mathrm{f}} k_{\text {cat }} / \mathrm{K}_{\mathrm{M}}$ \\
\hline Mut1 & $14.8 \pm 0.9$ & $0.21 \pm 0.01$ & $1.42 \pm 0.10$ & $3.0 \pm 0.3$ \\
Mut1_PGM & $11.0 \pm 0.7$ & $0.47 \pm 0.16$ & $4.26 \pm 0.32$ & \\
\hline Mut2 & $19.3 \pm 1.5$ & $0.31 \pm 0.01$ & $1.61 \pm 0.15$ & $1.2 \pm 0.1$ \\
Mut2_PGM & $10.8 \pm 0.7$ & $0.21 \pm 0.01$ & $1.90 \pm 0.14$ & \\
\hline Mut3 & $18.4 \pm 1.9$ & $0.05 \pm 0.00$ & $0.28 \pm 0.03$ & $2.9 \pm 0.4$ \\
Mut3_PGM & $14.8 \pm 1.5$ & $0.12 \pm 0.01$ & $0.81 \pm 0.08$ & \\
\hline Mut4 & $25.3 \pm 2.5$ & $0.71 \pm 0.04$ & $2.79 \pm 0.32$ & $4.1 \pm 0.5$ \\
Mut4_PGM & $7.9 \pm 0.5$ & $0.91 \pm 0.02$ & $11.4 \pm 0.74$ & \\
\hline Mut5 & $16.0 \pm 2.3$ & $0.13 \pm 0.01$ & $0.79 \pm 0.13$ & $0.9 \pm 0.1$ \\
Mut5_PGM & $12.4 \pm 0.5$ & $0.09 \pm 0.00$ & $0.72 \pm 0.04$ & \\
\hline Mut6 & $15.6 \pm 2.9$ & $0.15 \pm 0.01$ & $0.95 \pm 0.20$ & $7.9 \pm 1.9$ \\
\hline Mut6_PGM & $8.7 \pm 0.9$ & $0.65 \pm 0.02$ & $7.50 \pm 0.85$ & \\
\hline WT & $15.2 \pm 0.5$ & $0.17 \pm 0.00$ & $1.10 \pm 0.07$ & -
\end{tabular}

a The experimental conditions used here are different than in the previous report of mut6 28

${ }^{\mathrm{b}} \mathrm{K}_{\mathrm{M}}$ is expressed in $\mu \mathrm{M}$

${ }^{\mathrm{c}} k_{\text {cat }}$ is expressed in $\mathrm{s}^{-1} 10^{-3}$

${ }^{\mathrm{d}} k_{\text {cat }} / \mathrm{K}_{\mathrm{M}}$ is expressed in $\mu \mathrm{M}^{-1} \mathrm{~s}^{-1} 10^{-2}$

e Fold $k_{\text {cat }} / K_{M}$ represent the fold increase of the PGM labeled mutant versus unlabeled

Table 2. BFC debenzylation kinetic data of P450 3A4 WT and PGM labeled and unlabeled mutants. Standard error is given.

Bioconjugation of PGM to the mutants, however, gave rise to two different scenarios: 1) the PGM-bioconjugate mutant remained activated by PRG, as with mut3-5; or 2) PRG did not activate the bioconjugate anymore, as with mut1,2 and 6. In the latter case, as the concentration of activator is increased from 0 to $10 \mu \mathrm{M}$, the enzyme activity towards BFC remains unchanged (Figure 4), whereas it decreases at higher concentrations of PRG, as expected since PRG should start competing with BFC as the substrate. The absence of PRG activation for the bioconjugates of mut1, 2, and 6 is consistent with the PRG moiety of the PGM label residing in the allosteric pocket and therefore preventing free PRG from binding and further activating the enzyme. In the case of mut1 and mut6, the increase in activity of the bioconjugates compared to the unlabeled mutants suggests that the covalently attached PGM molecule may permanently activate the enzyme by binding to the PRG allosteric pocket. For the PGM-bioconjugate of mut2, the plateau observed at PRG concentrations below $10 \mu \mathrm{M}$ is consistent with the PGM moiety competing with free PRG, but the overall decrease in activity compared to that of the unmodified mutant suggest that although the position of PGM may prevent PRG binding at the allosteric site, its orientation or position in the pocket does not trigger activation. The fact that the PGM moiety is able to completely block activation by free PRG for mut1, mut2 and mut6 implies that only a single binding pocket exists for positive allosteric activation by PRG and that this allosteric pocket is located at a position that can be reached by the PGM labels of mut1, 2 and 6. On the other hand, the PGM-bioconjugates of mut35 remained activated by free PRG, consistent with the PGM label not residing in the allosteric pocket. Interestingly, the PGM bioconjugates of mut3 and mut4 showed an increased turnover of BFC compared to the unlabeled mutants even 
This document is the unedited Author's version of a Submitted Work that was subsequently accepted for publication in 'Bioconjugate Chemistry', copyright (C) 2019 American Chemical Society after peer review. To access the final edited and published work see https://doi.org/10.1021/acs.bioconjchem.9b00248

though the PGM moiety is not residing in the allosteric pocket. These results are interesting as they propose that other activation mechanisms may be involved.
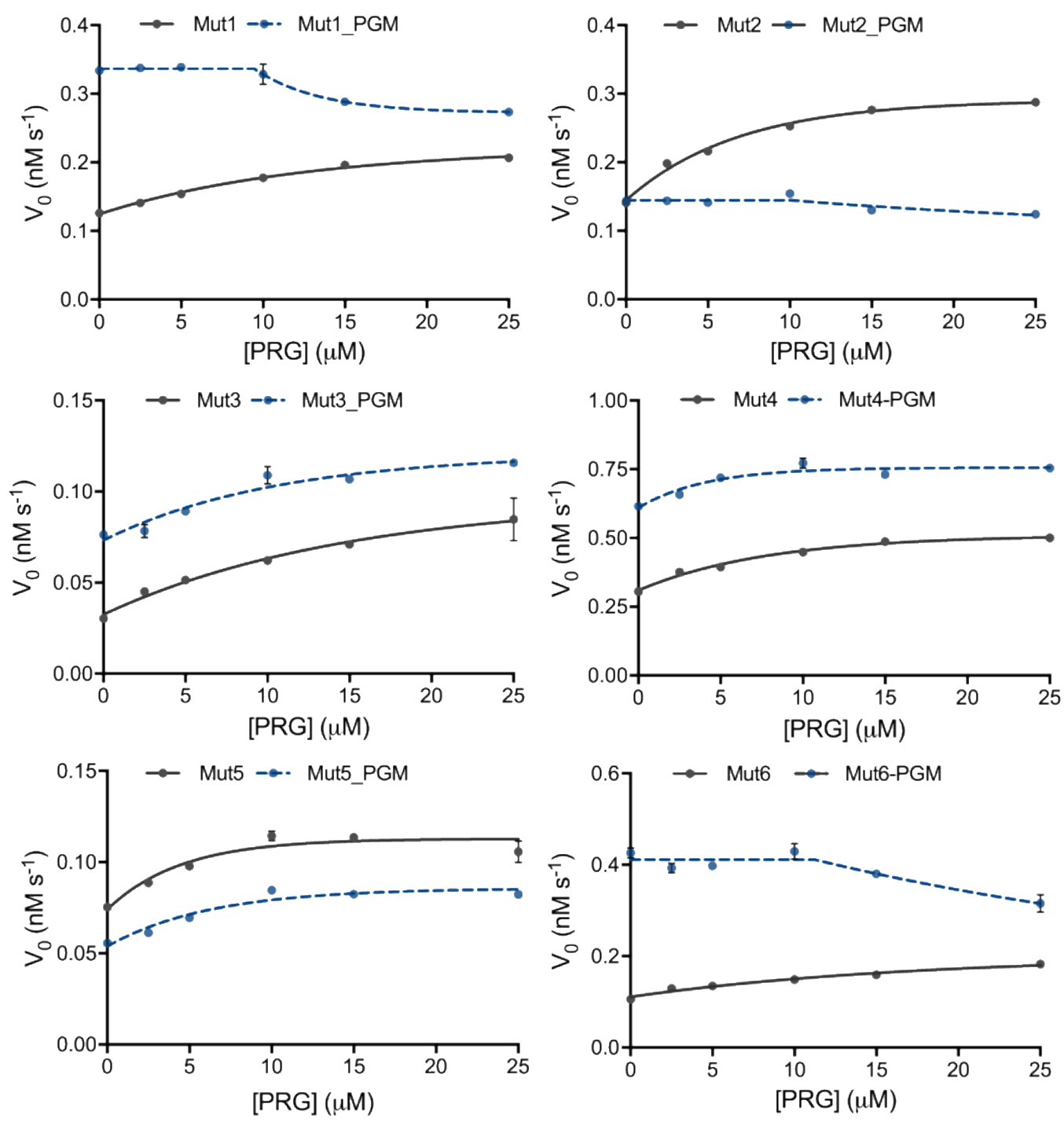

Figure 4. Progesterone activation assay comparison of PGM-labeled vs. unlabeled P450 3A4 mutants. The reaction mixtures consisted of $0.7 \mu \mathrm{M}$ P450, $0.7 \mu \mathrm{M}$ CPR, $50 \mu \mathrm{M}$ BFC, $0-25 \mu \mathrm{M}$ PRG and $1 \mathrm{mM}$ NADPH. The dotted blue curves represent the PGMlabeled mutants and the solid curves the unlabeled mutants. Each data point is the average of triplicates with the standard error shown with error bars.

\section{Location of the PRG allosteric site}

While kinetic data points to the PGM labels of mut1 and mut6 activating the enzyme, our PRG activation studies show that the label prevents free PRG from further activating the enzyme towards BFC debenzylation. Together this is 
This document is the unedited Author's version of a Submitted Work that was subsequently accepted for publication in 'Bioconjugate Chemistry', copyright (C) 2019 American Chemical Society after peer review. To access the final edited and published work see https://doi.org/10.1021/acs.bioconjchem.9b00248

consistent with the PGM moiety of the bioconjugates occupying the allosteric site in a position and orientation conducive to activation. The PGM label in mut2 also prevents free PRG from binding the allosteric site but did not enhance BFC debenzylation. This suggests that the PGM moiety of the mut2 bioconjugate prevents free PRG from binding without mimicking allosteric activation.

By building the PGM molecule directly into the PRG-CYP3A4 crystal structure (PDB: 1WOF), we evaluated, based on the PGM attachment point (cysteine), if the PGM moiety of the bioconjugates could align with the co-crystallized progesterone molecule. The objective was to assess if the PGM label in mut1, mut2, and mut6 could reach the putative allosteric pocket observed in the crystal structure of the PRG-CYP3A4 complex (Figure 5). Interestingly, for mut6 (F215C), the most PGM-activated bioconjugate, the PGM can align almost perfectly with the co-crystallized PRG molecule. For the bioconjugate of mut1 (F108C), the second most activated, the PGM moiety can orient similarly, but not exactly like the PRG in the crystal structure. Indeed, F215 and F108 are located at the same end of the allosteric pocket, but on loops facing each other, the $\mathrm{B} / \mathrm{B}^{\prime}$ and $\mathrm{F} / \mathrm{F}^{\prime}$ loops. This implies that small changes in the binding geometry of PRG can alter the magnitude of the activation and shows that slightly different binding orientations of PRG in the allosteric pocket all leading to enzyme activation. Finally, the PGM moiety of the mut2 bioconjugate, which decreased BFC debenzylation compared to the unlabeled mut2, was only able to partially reach the putative allosteric site, possibly explaining its antagonistic behavior (i.e. bioconjugation leading to decreased $k_{\text {cat }}$; Table 2, Figure 4).
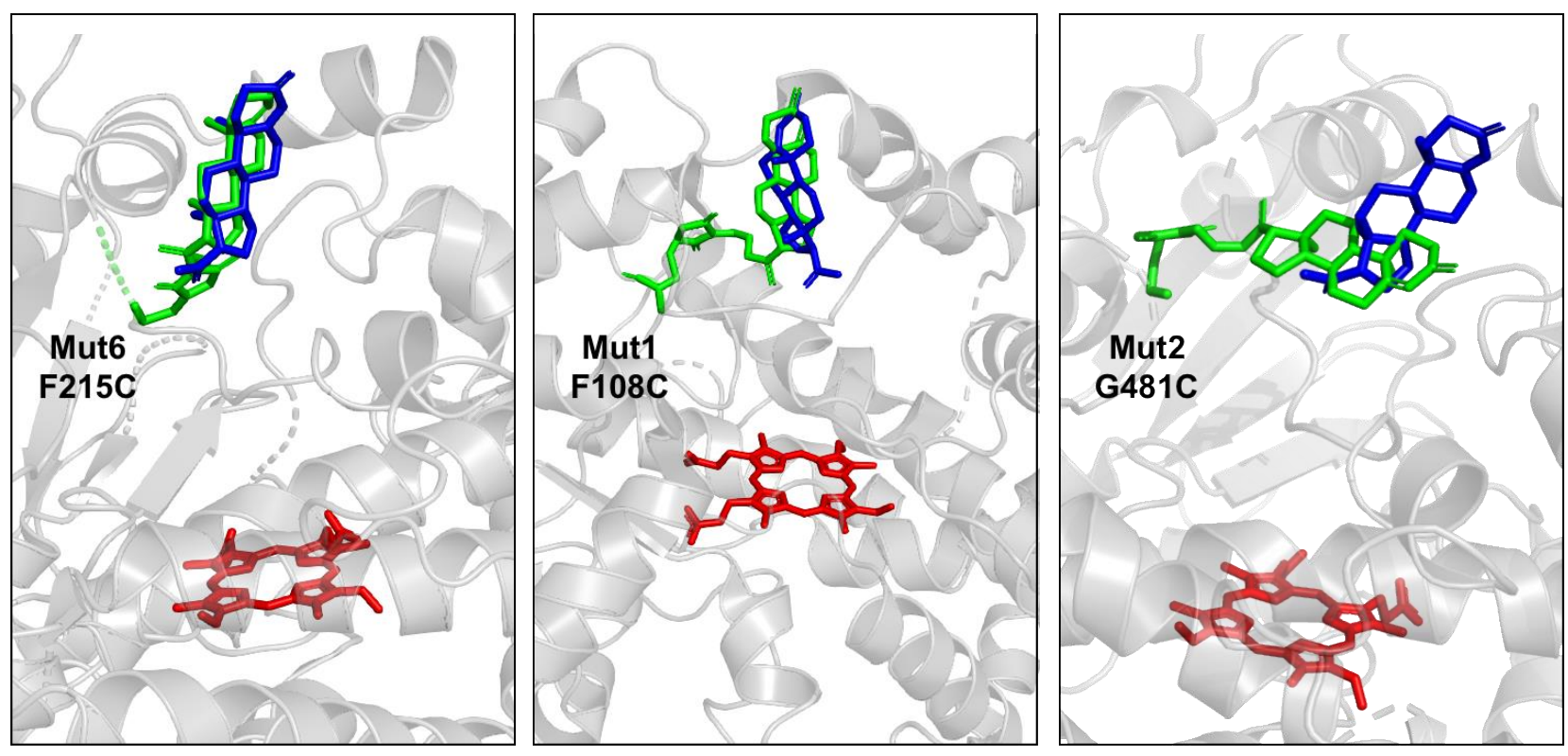

Figure 5. Possible binding orientation of the PGM label in mut6 (F215C), mut1 (F108C) and mut2 (G481C) bioconjugates. The figure shows the PGM label in green, the heme in red and the co-crystallized PRG in blue. The PGM groups were built directly in the PDB structure and positioned as much as possible to reach the same orientation as the co-crystallized PRG (PDB: 1WOF).

The combination of our kinetic and PRG activation data, together with the proposed PGM-CYP3A4 structures (Figure 5), strongly suggest that the PGM labels of mut1 and mut6 lie in the same pocket as free PRG. Our data however 
This document is the unedited Author's version of a Submitted Work that was subsequently accepted for publication in 'Bioconjugate Chemistry', copyright (C) 2019 American Chemical Society after peer review. To access the final edited and published work see https://doi.org/10.1021/acs.bioconjchem.9b00248

does not indicate the precise location of this pocket, and it may be different from that seen in the co-crystalized PRG in the CYP3A4 crystal structure. The PGM label could, for example, point inside the active site rather than in the allosteric pocket as shown in Figure 5. A recent paper by Hackett explored the different modes of binding of testosterone in CYP3A4 through computational studies. ${ }^{31}$ The authors identified a novel temporary binding site for testosterone away from the heme. Looking at their 3D structures of the enzyme, our mutation sites, and the geometry of PGM, it is unlikely that the PGM moiety of our mut1 and mut6 bioconjugates would sit in this temporary binding area in the proper orientation (Figure S9). This suggests that the reported transient testosterone binding site is different from the PRG allosteric pocket.

\section{Specificity of PRG activation}

Since the kinetics of CYP3A4-catalyzed BFC debenzylation exhibits positive cooperativity (in the absence of PRG, Figure S10), we expected that coumarin bioconjugation might also lead to enhancement of BFC debenzylation. In order to investigate this, we covalently attached coumarin-maleimides DACM and MDCC - which differ mostly by the presence of a 2-carbon linker between the coumarin and the maleimide group (Figure S3) - separately to mut1-5 (Figure S11a-c). Bioconjugation of DACM had a detrimental effect on the activity of most mutants towards BFC. On the other hand, MDCC bioconjugation had little effect on mut1-3, but increased the activity of mut4-5 towards BFC (Figure S11d). These results suggest that the coumarin moiety of the mut 4 and mut5 bioconjugates may reach the BFC allosteric site, although further studies are warranted to confirm this. Considering the location of residues V111 (Cys in mut4) and Q484 (Cys in mut5) in CYP3A4 (Figure 2) and the length of MDCC, it is unlikely that the coumarin ring of MDCC would be able to occupy the PRG allosteric site, implying that the allosteric pockets of PRG and BFC may be different. This also suggests that the mechanism underlying BFC homotropic cooperativity most likely differs from the PRG-driven activation of CYP3A4 during BFC debenzylation.

\section{Conclusion}

We have demonstrated that bioconjugation of PGM at the allosteric site of CYP3A4 can mimic the allosteric activation of BFC debenzylation by free PRG. Among the different PGM bioconjugates reported here, those of mut1 and mut6 were the only ones that replicated allosteric activation by PRG. By comparing the PRG activation profiles of different bioconjugates, we were able to show that the PGM groups of mut1 and mut 6 both occupy the same pocket as free PRG. We can therefore narrow down the position of the PRG allosteric site. Moreover, the excellent overlap of the PGM labels with the co-crystallized PRG in CYP3A4 suggests that this PRG peripheral binding site may be an allosteric site. Interestingly, the activity of the PGM bioconjugate of mut2 was attenuated relative to the respective unmodified mutant, and was not activated by free PRG, implying that partial occupation of the allosteric site may antagonize with PRG allostery in this bioconjugate. Overall, the bioconjugation of a PRG analogue to CYP3A4 proved to be an effective approach for the investigation of heterotropic cooperativity and offers further opportunities to study other allosteric systems. 
This document is the unedited Author's version of a Submitted Work that was subsequently accepted for publication in 'Bioconjugate Chemistry', copyright (C) 2019 American Chemical Society after peer review. To access the final edited and published work see https://doi.org/10.1021/acs.bioconjchem.9b00248

Supporting Information. Material and methods, mutants BFC activity, bioconjugation reaction yields, absorbtion spectra of PGM-bioconjugates, mutant sequence primers and supplemental figures described in the text. The Supporting Information is available free of charge on ACS Publications website.

Acknowledgements. This research was funded by the National Science and Engineering Research Council of Canada (NSERC) and by the Center in Green Chemistry and catalysis (CGCC). Julie Ducharme was supported by scholarships from the CGCC and the Fonds de recherche du Quebec - nature et technologies (FRQNT). Vanja Polic was supported by scholarships from the Dr. Richard H. Tomlinson Foundation, Walter C. Sumner Foundation, the CGCC, and NSERC. We would like to thank Dr. A. S. Wahba for his work on protein LC-MS-QToF, Dr. J. R. Halpert for providing the CYP3A4 plasmid and Dr. C. B. Kasper for the CPR plasmid. Lastly, we would like to thank Dr. Hackett for kindly sharing his 3D structure of CYP3A4 with a testosterone molecule positioned in a putative temporary binding site.

\section{$\underline{\text { References }}$}

(1) Nussinov, R.; Tsai, C.-J.; Ma, B. The (Still) Underappreciated Role of Allostery in the Cellular Network. Annu Rev Biophys 2013, 35, 169-189.

(2) Motlagh, H. N.; Wrabl, J. O.; Li, J.; Hilser, V. J. The Ensemble Nature of Allostery. Nature 2014, 508 (7496), $331-339$.

(3) Guengerich, F. P. Cytochrome P-450 3A4: Regulation and Role in Drug Metabolism. Annu. Rev. Pharmacol. Toxicol. 1999, 39, 1-17.

(4) Ekroos, M.; Sjogren, T. Structural Basis for Ligand Promiscuity in Cytochrome P450 3A4. Proc. Natl. Acad. Sci. 2006, 103 (37), 13682-13687.

(5) Domanski, T. L.; He, Y.; Harlow, G. R.; Halpert, J. R. Dual Role of Human Cytochrome P450 3 A4 Residue Phe-304 in Substrate Specificity and Cooperativity 1. 2011, 293 (2), 585-591.

(6) Ueng, Y.-F.; Kuwabara, T.; Chun, Y.-J.; Guengerich, F. P. Cooperativity in Oxidations Catalyzed by Cytochrome P450 3 A4 †. 1997, 36 (2), 370-381.

(7) Domanski, T. L.; He, Y. A.; Khan, K. K.; Roussel, F.; Wang, Q.; Halpert, J. R. Phenylalanine and Tryptophan Scanning Mutagenesis of CYP3A4 Substrate Recognition Site Residues and Effect on Substrate Oxidation and Cooperativity. Biochemistry 2001, 40 (34), 10150-10160.

(8) Frank, D. J.; Denisov, I. G.; Sligar, S. G. Mixing Apples and Oranges: Analysis of Heterotropic Cooperativity in Cytochrome P450 3A4. Arch. Biochem. Biophys. 2009, 488 (2), 146-152. h

(9) Hutzler, J. M.; Tracy, T. S. Atypical Kinetic Profiles in Drug Metabolism Reactions. Drug Metab. Dispos. 2002, 30 (4), 355362.

(10) Johnson, E. F.; Schwab, G. E. Constitutive Forms of Rabbit-Liver Microsomal Cytochrome P-450: Enzymatic Diversity, Polymorphism and Allosteric Regulation. Xenobiotica 1984, 14 (1-2), 3-18.

(11) Johnson, E. F.; Schwab, G. E.; Vickery, L. E. Positive Effectors of the Binding of an Active Site-Directed Amino Steroid to Rabbit Cytochrome P-450 3c. J. Biol. Chem. 1988, 263 (33), 17672-17677.

(12) Raney, K. D.; Shimada, T.; Kim, D. H.; Groopman, J. D.; Harris, T. M.; Guengerich, F. P. Oxidation of Aflatoxins and Sterigmatocystin by Human Liver Microsomes: Significance of Aflatoxin Q1 as a Detoxication Product of Aflatoxin B1. Chem. Res. Toxicol. 1992, 5 (2), 202-210.

(13) Williams, P. A.; Cosme, J.; Vinkovic, D. M.; Alison, W.; Angove, H. C.; Day, P. J.; Clemens, V.; J.T, I.; Jhoti, H. Crystal Structures of Human Cytochrome P450 3A4 Bound to Metyrapone and Progesterone. Science (80-. ). 2004, 305 (5684), $683-686$.

(14) Davydov, D. R.; Rumfeldt, J. A. O.; Sineva, E. V.; Fernando, H.; Davydova, N. Y.; Halpert, J. R. Peripheral Ligand-Binding Site in Cytochrome P450 3A4 Located with Fluorescence Resonance Energy Transfer (FRET). J. Biol. Chem. 2012, 287 (9), 67976809.

(15) Marsch, G. A.; Carlson, B. T.; Guengerich, F. P. 7,8-Benzoflavone Binding to Human Cytochrome P450 3 A4 Reveals Complex Fluorescence Quenching, Suggesting Binding at Multiple Protein Sites. J. Biomol. Struct. Dyn. 2018, 36 (4), $841-860$.

(16) Roberts, A. G.; Atkins, W. M. Energetics of Heterotropic Cooperativity between A-Naphthoflavone and Testosterone 
This document is the unedited Author's version of a Submitted Work that was subsequently accepted for publication in 'Bioconjugate Chemistry', copyright (C) 2019 American Chemical Society after peer review. To access the final edited and published work see https://doi.org/10.1021/acs.bioconjchem.9b00248

Binding to CYP3A4. Arch. Biochem. Biophys. 2007, 463 (1), 89-101.

(17) Society, A. C.; Id, M.; Type, M.; Submitted, A. D.; List, C.; Grinkova, B.; Nandigrami, B.; Shekhar, B.; Science, A.; Tajkhorshid, T. Allosteric Interactions in Human Cytochrome P450 CYP3A4 : The Role of Phenylalanine 213. Biochemistry 2019, 58 (10), 1411-1422.

(18) Sineva, E. V.; Rumfeldt, J. A. O.; Halpert, J. R.; Davydov, D. R. A Large-Scale Allosteric Transition in Cytochrome P450 3A4 Revealed by Luminescence Resonance Energy Transfer (LRET). PLoS One 2013, 8 (12), e83898.

(19) Roberts, A. G.; Campbell, A. P.; Atkins, W. M. The Thermodynamic Landscape of Testosterone Binding to Cytochrome P450 3A4: Ligand Binding and Spin State Equilibria. Biochemistry 2005, 44 (4), 1353-1366.

(20) Isin, E. M.; Guengerich, F. P. Kinetics and Thermodynamics of Ligand Binding by Cytochrome P450 3A4. J. Biol. Chem. 2006, 281 (14), 9127-9136.

(21) Ichikawa, T.; Tsujino, H.; Miki, T.; Kobayashi, M.; Matsubara, C.; Miyata, S.; Yamashita, T.; Takeshita, K.; Yonezawa, Y.; Uno, T. Allosteric Activation of Cytochrome P450 3A4 by Efavirenz Facilitates Midazolam Binding. Xenobiotica 2017, 48 (12), 1227-1236.

(22) Denisov, I. G.; Baylon, J. L.; Grinkova, Y. V.; Tajkhorshid, E.; Sligar, S. G. Drug-Drug Interactions between Atorvastatin and Dronedarone Mediated by Monomeric CYP3A4. Biochemistry 2018, 57 (5), 805-816.

(23) Denisov, I. G.; Mak, P. J.; Grinkova, Y. V.; Bastien, D.; Bérubé, G.; Sligar, S. G.; Kincaid, J. R. The Use of Isomeric Testosterone Dimers to Explore Allosteric Effects in Substrate Binding to Cytochrome P450 CYP3A4. J. Inorg. Biochem. 2016, 158, 77-85.

(24) Davydov, D. R.; Davydova, N. Y.; Sineva, E. V.; Halpert, J. R. Interactions among Cytochromes P450 in Microsomal Membranes: Oligomerization of Cytochromes P4503A4,3A5, and 2E1 and Its Functional Consequences. J. Biol. Chem. 2015, 290 (6), 3850-3868.

(25) P, C.; Nath, A.; Fernández, C.; Lampe, J. N.; Atkins, W. M. Spectral Resolution of a Second Binding Site for Nile Red on Cytochrome P450 3A4. Biochem. J. 2009, 474 (1), 198-204.

(26) Zhang, Y.-Y.; Yang, L. Interactions between Human Cytochrome P450 Enzymes and Steroids: Physiological and Pharmacological Implications. Expert Opin. Drug Metab. Toxicol. 2009, 5 (6), 621-629.

(27) Renwick, A. B.; Surry, D.; Price, R. J.; Lake, B. G.; Evans, D. C. Metabolism of 7-Benzyloxy-4-Trifluoromethylcoumarin by Human Hepatic Cytochrome P450 Isoforms. Xenobiotica 2000, 30 (10), 955-969.

(28) Polic, V.; Auclair, K. Allosteric Activation of Cytochrome P450 3A4 via Progesterone Bioconjugation. Bioconjug. Chem. 2017, 28 (4), 885-889.

(29) Polic, V.; Sevrioukova, I. F.; Auclair, K. Steroid Bioconjugation to a CYP3A4 Allosteric Site and Its e Ff Ect on Substrate Binding and Coupling Efficiency. 2018, 653 (May), 90-96.

(30) Ménard, A.; Huang, Y.; Karam, P.; Cosa, G.; Auclair, K. Site-Specific Fluorescent Labeling and Oriented Immobilization of a Triple Mutant of CYP3A4 via C64. Bioconjug. Chem. 2012, 23 (4), 826-836.

(31) Hackett, J. C. Membrane-Embedded Substrate Recognition by Cytochrome P450 3A4. J. Biol. Chem. 2018, 293 (11), 40374046.

\section{Graphical Abstract}


This document is the unedited Author's version of a Submitted Work that was subsequently accepted for publication in 'Bioconjugate Chemistry', copyright (C) 2019 American Chemical Society after peer review. To access the final edited and published work see https://doi.org/10.1021/acs.bioconjchem.9b00248

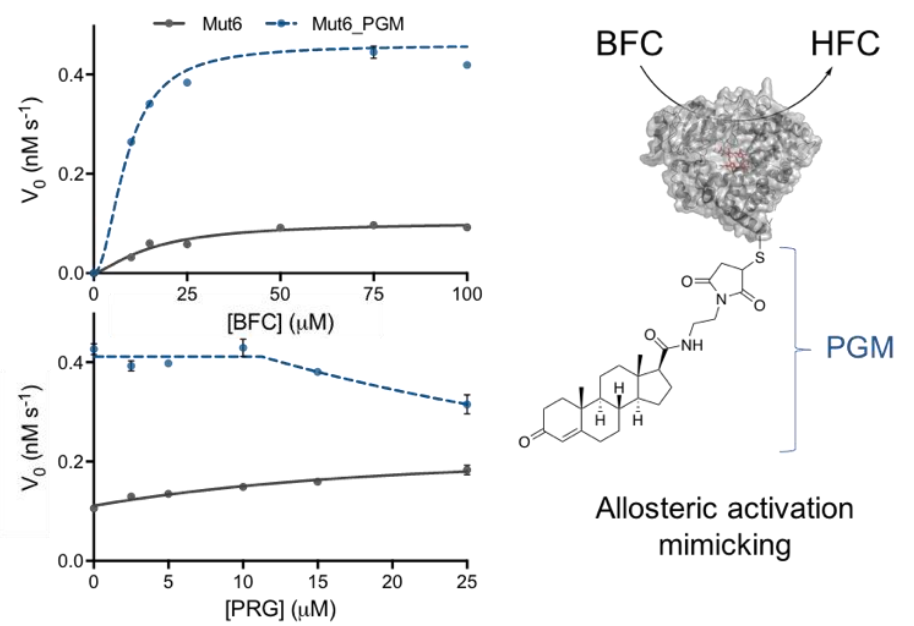

